\title{
Screen of Chryseobacterium CHANGBAl-2 strain and Identification of the Antifungal Antibiotic from Its Fermentation Broth
}

\author{
Ning YANG ${ }^{1}$, Yong WANG ${ }^{1, a^{*}}$, Yu-bo WANG ${ }^{2}$, Li ZHANG ${ }^{1}$, Yong LU $^{1}$, \\ Quan-jie CHEN ${ }^{1}$, Wen-ge $\mathrm{ZHANG}^{3}$, and Ming-shan $\mathrm{JI}^{2}$ \\ ${ }^{1}$ School of Chemical Engineering, University of Science and Technology Liaoning, \\ Anshan, 114051 P. R. China; \\ ${ }^{2}$ Shenyang Agricultural University, Shenyang, 110161 P. R. China \\ ${ }^{3}$ Anshan Normal University Affiliated Medical School, Anshan, 114001 P. R. China \\ awy2002866@126.com \\ ${ }^{*}$ Corresponding author
}

Keywords: Chryseobacterium, Screen, Antibiotic, identification, Fermentation broth

\begin{abstract}
CHANGBAI-2 actinomycete strain was obtained by separating different kinds of soils from the nature. Moreover, the morphology, cultural characteristics, physiological-biochemical characteristics and 16S rDNA sequences of this strain were studied. A phylogenetic tree was constructed by comparing with the published $16 \mathrm{~S}$ rDNA sequences of the related species and showed $99 \%$ identity of nucleotide sequence of $16 \mathrm{~S}$ rDNA with Chryseobacterium. The antifungal substance produced by CHANGBAI-2 was purified by means of macro-porous adsorbent resin, and the crystal of the antibiotic with high purity was got. In this paper, the methods of purification by adsorbing of microporous adsorbent resin and detection by high performance liquid chromatography with mass spectrum (HPLC-MS) were established. The study would be laid a good foundation for its physical-chemical properties.
\end{abstract}

\section{Introduction}

Actinomycetes is a kind of prokaryota which shape is hyphal. They mainly reproduce by spores and it's terraneous strongly. Its species is numerous; metabolic functions is different and it is a kind of biological resources which has wide application. About $70 \%$ of antibacterial substances in the category come from actinomycetes (Actinomycetes) [1]. Using a life-organisms and their metabolic characteristics of secretions, replicate (biological source) by the processing of the bactericidal function of pest control agents-antibiotics. Nowdays more and more countries pay their attentions to them because of their lower toxicity, high efficiency, strong selectivity, short residual time and pollution-free.

In this paper, screen actinomycetes and identify strain collected from different soils. We explore the technology about isolation and purification of potent antimicrobial activity made by the Chryseobacterium. Moreover, identify molecular structure of CHANGBAI-2 initially. All of the works enrich their theoretical foundations which could further explore its mechanism and also lay the foundations for further amplification and mass production. 


\section{Experimental}

\section{Materials}

The samples. The soil samples are collected from different elevations, different vegetation, and different soil types and the water samples.

Culture Medium. PDA medium, GAU medium, GAR medium, GAS medium, EM medium, SC medium, KB medium.

Macroporous Resin. CAD-45, middle polarity.

Thin-layer Solvent. G254 (200 300 section).

Main instruments and reagents. Light microscope, Electron microscope, polymerase Chain Reaction(PCR) amplification machine, Nucleic acid electrophoresis apparatus, Protein electrophoresis apparatus, DNA sequencing machie, BigDye Terminator V3.1 Cycle Sequencing Kit, High speed centrifuge, Constant temperature shaker. Glass adsorbing column; LXJD ls high-capacity centrifuge; Freezer dryer; LAMBDA900 ultraviolet spectrophotometer; 500 trillion Nuclear Magnetic Resonance Spectrometer; WQF200 Fourier infrared spectroscopy; High Performance Liquid Chromatography (HPLC) / mass-spectrograph.

\section{Methods}

Isolation and Screening of Actinomycetes Strains. The strains are isolated with the method of dilution. While separating actinomycetes strains from soil, we should first make the soil samples dried at room temperature for $10 \mathrm{~d}$ in order to prevent other miscellaneous bacteria pollution. After that it can be used to prepare soil suspension. Under the sterile condition, imbibe $0.1 \mathrm{ml}$ suspension into GAU medium. Spread the plate uniformly with sterile spreader. Upside down the culture plate at $28^{\circ} \mathrm{C}$ for $10--15 \mathrm{~d}$.

Identification of Bacterial Strain. (1)morphological characteristics. Using male tab method, after 7 day's cultivation at $28^{\circ} \mathrm{C}$, view the morphologic characteristics of aerial hypha and spore with light microscope and electrical microscope. (2)Cultural characteristics. According to the partial medium referred by the streptomycete identification guide [2], culture the strains for $168 \mathrm{~h}$ at $28^{\circ} \mathrm{C}$. Then observe and record the change of color, state and pigment. (3) Physiological and biochemical detection. Refer to streptomycete identification guide, gelatin liquefaction of the strain CHANGBAI-2, and the detection also contains solidfication of milk, Starch hydrolysis, cellulose decomposition, nitrate reduction. It also includes the production of hydrogen sulfide, utilization of carbon source and production of melanin and other physiological and biochemical detections.

The Squencing and Analysis of 16S rDNA. Extract total DNA from fresh strains cultured in plate using microwave method [3]. Then amplify 16sDNA. Prepare primer according to the structural features and conserved rigion of 16sDNA.

Construction of Phylogenetic Tree. First, use the BLAST software to carry on comparison in the GenBank database for the 16SrDAN sequence of a typical strains which are similar to the experiment strain, then we selected 16S rDAN sequence of typical Streptomyces about 12 strains as outgroup, and arrange the target sequence by the DNAstar software. Take the Boot-strapping method to assess constructed evolutionary tree.

The Extracts of Antimicrobial Activity. Put fermented fluid of CHANGBAI-2 about $150 \mathrm{ml}$ into the macro-porous adsorbent resin $(10 \mathrm{~cm} \times 100 \mathrm{~cm}$ the pillars of glass $)$ and then choose different concentrations of ethanol as eluant which elution volume is 
$500 \mathrm{ml}$ in order to pick out the most suitable eluant of concentration. Colecting fraction $10 \mathrm{ml} /$ tube, then respectively detect its antimicrobial activity. Add activated charcoal into active eluant components. Take steam from the miscible and frozen, dry. Finally get coarse crystallization $[4,5]$.

Purification of Antibacterial Substances. Adopt the thin layer chromatography method, inhibitory effects were obtained for further purification of the crude fruit of the best. Take a small amount of resin obtained by the large aperture of the antibacterial activity of crude material, dissolve with dichloromethane, pipette $1.0 \mathrm{ml}$ solution, spot in a straight line on the $10 \mathrm{~cm} \times 20 \mathrm{~cm} \times 0.2 \mathrm{~cm}$ Thin-layer chromatography (TLC) plate $2 \mathrm{~cm}$ far from the bottom edge, place them in the surface active agent of petroleum ether: ethyl acetate $(5: 1, \mathrm{v} / \mathrm{v})$, making a layer of vertical development (Make a determination after comparing the effects that the polarity of the surface active agent have on the results of chromatographic),make a detection with ZF7B type three Ultraviolet(UV) analyzer at $527 \mathrm{~nm}$ UV light, determine the band, measure and calculate the relative mobility of bands. Choose the active component that across TLC silica gel plate, scrape the component that show a single component on the plate, shake and immerse with dichloromethane, extracting $6 \mathrm{~h}$. Then filter the gel, volatile the solvent, draine with a vacuum freeze-drying machine, and the puring active antibacterial substances can be obtained.

The Preliminary Analysis of the Structure of the Active Antibacterial Substances. The purity was analysed by HPLC-MS (the Agilent Company of USA). Desolve the samples that have acrossed large diameter resin and TLC with methanol chromatography, adjust the concentration to $1 \times 10^{3} \mu \mathrm{g} / \mathrm{mL}$, then it is sample solution after acrossing the microporous membrane(Organic phase $0.45 \mu \mathrm{m})[6,7]$. Chromatographic conditions: Chromatographic column Eclipse Plus C18, $150 \mathrm{~mm} \times 4.6 \mathrm{~mm}$, the mobile phase is methanol, velocity of flow $0.8 \mathrm{~mL} / \mathrm{min}$, column temperature: $30^{\circ} \mathrm{C}$; Detector: Diode array detector (DAD); Wavelength: $232 \mathrm{n}$, Bandwidth: 4. MS conditions: Ion source, atmospheric chemical ionization (APCI) ; Polarity: Positive; Drying temperature: $325^{\circ} \mathrm{C}$; Ion source temperature: $400^{\circ} \mathrm{C}$; Atomization air pressure: 60.0 psi; Drying gas flow rate:5.0 L/min; Scan range $(\mathrm{m} / \mathrm{z})$ : $100 \sim 900$, full scan. Preliminary analysis of the structure is the combination of various spectral analysis method.

\section{Results and Analysis}

Screening of Bacterial Strain. The pure CHANGBAI-2 strain of actinomycetes was isolated from different soil, water samples at different altitudes from the nature, as is shown in the figure1.
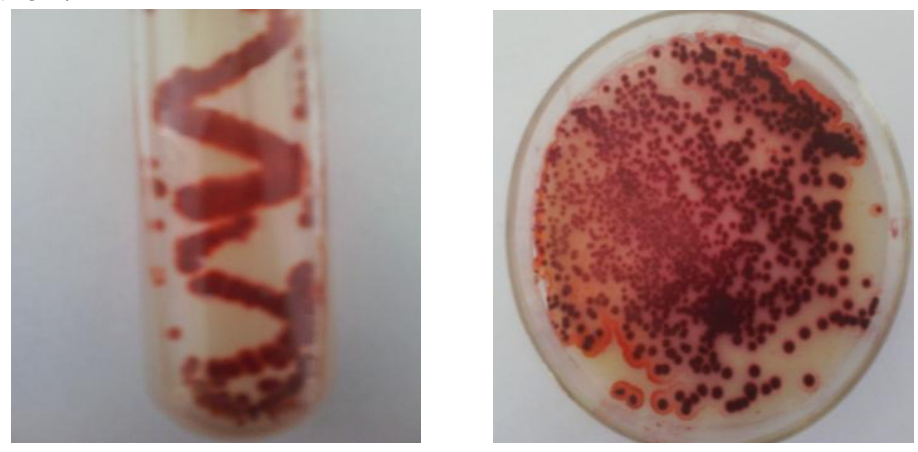

Fig.1 Actinomycetes separated from soil sample 
Morphology Observation of Antagonistic Strain CHANGBAI-2. By optical microscopy and electron microscopy revealed (shown in the Figure 2): The basal filament of CHANGBAI-2 is not broken, no diaphragm, composition branched; spores is round to oval and its surface is smooth. Sporothrix is loose ring, primary spiral, or hook.
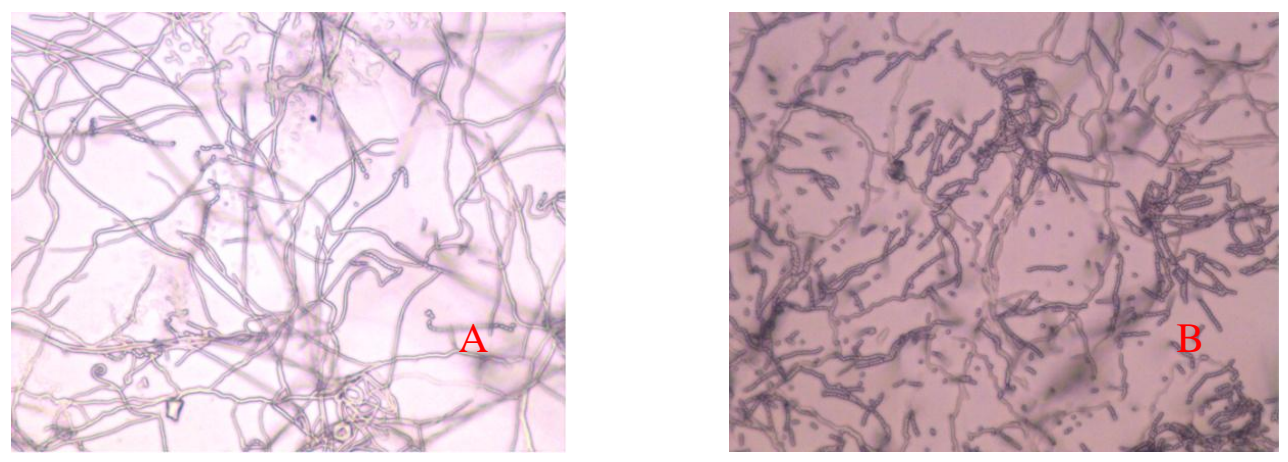

Fig.2 Morphologic characters of mycelium of strain CHANGBAI-2 (A: substrate mycelium B: Sporothrix)

The Physiological and Biochemical Characteristics of CHANGBAI-2 Strain and its Cultivation Characteristics. The culture results of CHANGBAI-2 strains in different media: observed in the conventional culturing method, CHANGBAI-2 strains of actinomycetes grow well in GAU, Glucose-asparagine prime medium, potato decoction, EM, Czapek Sucrose, inorganic salts starch this six mediea, its colonies is white, opaque and has a dense velvet-like surface. Its aerial mycelia is gray, substrate mycelium is white, and does not produce soluble pigments. The results of the physiological and biochemical measurements show that CHANGBAI-2 strains of actinomycetes are gram-positive; gelatin liquefaction; milk coagulation; cannot hydrolyze starch; can hydrolyze cellulose; nitrate reduction positive; cannot produce melanin and $\mathrm{H}_{2} \mathrm{~S}$; be able to use glucose, fructose, xylose, inositol, sucrose, rhamnose, mannitol.

The Molecular Identification of CHANGBAI-2. The similarity analysis of BLAST indicates that the Sequence homology of 16S rDNA between actinobacteria CHANGBAI-2 strains and Chryseobacteriumsp.PSB1-20 reachs 99\%. Actinobacteria CHANGBAI-2 strains and the relevant strains of phylogenetic tree (shown in the Figure 3). So we can infer: actinobacteria CHANGBAI-2 strains and Chryseobacterium belong to the same branch.

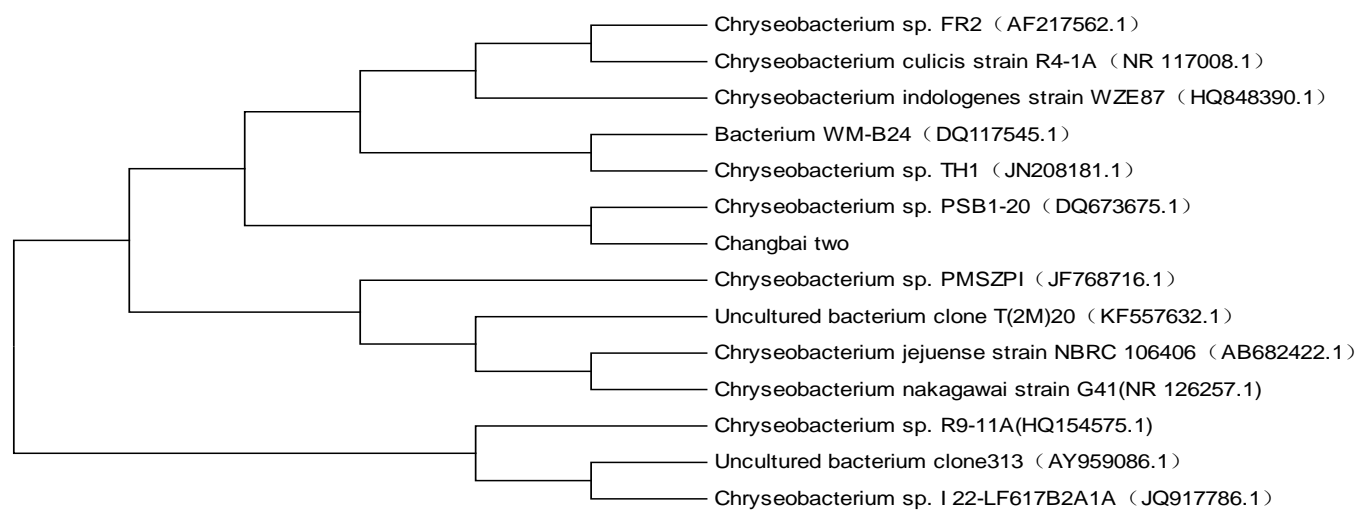

Fig.3 Phylogenetic tree of strain CHANGBAI-2 and related strains based on 16S rDNA 
The Research of Macroporous Resin Static Attaching Antimicrobial Activity Material. Select the adsorbent (non-polar CAD-45) which is suitable for antibiotic separation and has broader adsorbent polarity, meanwhile to ethanol as a common analytical agent, antimicrobial active ingredient of its adsorption capacity in Table1. $30 \%$ ethanol as eluent separated the activity of the antibacterial component is high, a significant difference with the control. Therefore, $30 \%$ ethanol was chosen as the resolution agent.

Tab.1 Results of separating antimicrobial substanes from actinomycetes strain CHANGBAI-2 by macroporous adsorption resin

\begin{tabular}{cccc}
\hline & \multicolumn{2}{c}{ Solvent } & \\
\cline { 2 - 3 } Number & Kinds & Concentration & $\begin{array}{c}\text { Inhibitory diameter } \\
(\mathrm{mm})\end{array}$ \\
\hline 1 & Ethanol & $70 \%$ & $30.0 \mathrm{Bc}$ \\
2 & Ethanol & $50 \%$ & $24.7 \mathrm{ABb}$ \\
3 & Ethanol & $30 \%$ & $31.0 \mathrm{Aa}$ \\
4 & Control & $0 \%$ & $14.7 \mathrm{Aa}$ \\
\hline
\end{tabular}

Therefore, the macro-porous resin column with $30 \%$ ethanol was eluted, and then collected the eluent with segmentation, and measured the antibacterial activity of the eluent. For the collected eluent before and after $200 \mathrm{~mL}$, there was no antibacterial activity. The diameter of indicative bacteria's inhibition zone about the eluent collected from 8-18 units were 30mm or more, meaning a good separation effect on the elution of this section, so these eluent were concentrated and purified. Only few active substances from other parts of the eluent were isolated, so it can be discarded.

\section{Results on Separation of the Crude Extracts of the Antibacterial Active} Substances by Simulated Moving Bed (SMB)

The crude extracts obtained from macro-porous resin were further purified by a self-made three-zoned SMB. And then the final product was got with the yield of $45 \%$. The percentage content of the final product of $98 \%$ was investigated with the help of HPLC, (shown in the Figure 4).

\section{Structure Determination of Antibacterial Active Substance}

The antibacterial active substance which was separated and purified by SMB could be named as Changbai-1-II. Its structure was analyzed by UV, Infrared Radiation(IR) and MS. According to the positive ion mode-electrospray ionization-mass spectrometry (shown in the Figure 5). 


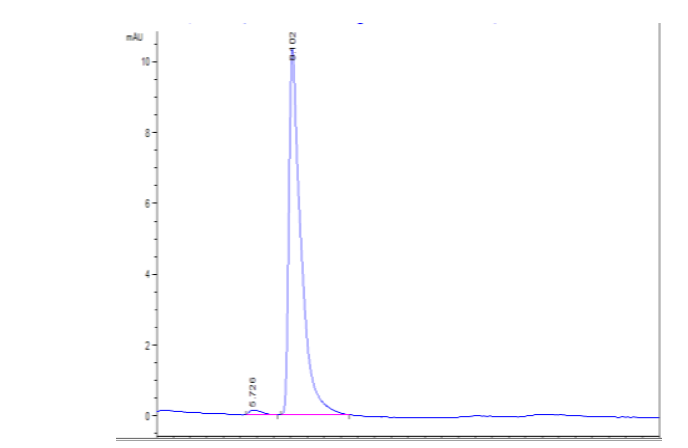

Fig. 4 Chromatogram of chromatographic product CHANGBAI- 2 by macroporous resin Extraction by SMB

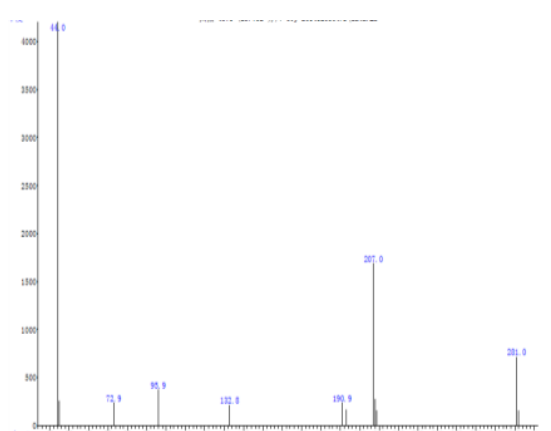

Fig.5 ESI-MS $[\mathrm{M}+\mathrm{H}]^{+}$spectrum of changbai-1-II Inset: its chemical structure

\section{Conclusion and Discussion}

Generally speaking, when two bacterial strains' homology of gene sequence of $16 \mathrm{~S}$ rRNA is less than $97 \%$, they are of different kinds; but when it is more than $97 \%$, they may also be different kinds. In this study, 27f/1510r is used as primers. Through the method of PCR gene amplification, full-length sequence, 1475bp, of 16S rRNA of actinomyces CHANGBAI-2 strains is obtained. By sequence analysising and constructing phylogeny trees, from the higher homology of bacterial strain or phylogeny trees, it can be seen that the higher homology to strains of actinomycete CHANGBAI-2 is Chryseobacteriumsp. Chryseobacteriumsp $16 \mathrm{~S}$ rDNA is in the same branch with actinomyces CHANGBAI-2 strains, and the homology is more than 97\%. But in the morphological characteristics, cultivating characteristics and physiological and biochemical characteristics, there are some differences. At present, it is still not sure whether the strain is a new species.

\section{References}

[1] Canedo L M,Puents J L F, Perez B J,et a1.IB-96212,a novel cytotoxic Macrolide produced by a marine Micromonospora.II.Phsico-chemical properties and structure determination [J].J Antibiot, 2000;53(5):479.

[2] Institute of Microbiology, Chinese Academy of taxonomic groups of actinomycetes. Streptomyces identification manual. Beijing: Science Press, 1975, 13-15. (In Chinese)

[3] XuPing, Li Wen-Jun Li,Xu Li-Hua, et al. MICROWAVE method for rapid extraction of actinomycetes genome DNA [J]. Microbiology, 2003, 30 (4): 82-84. (In Chinese)

[4] Gillespie,D.E., Brady,S.F., Bettermann,A.D. et al.Isolation of antibiotics turbomycin A and B from a metagenome library of soil microbial DNA. Appl. Environ.Microbiol., 2002, 68: 4301-4306.

[5] Ogawara,H., Kawamura,N., Kudo,T.et al.,Distribution of $\beta$-lactamases in actinomycetes.Antimicrob.Agents.Chemother., 1999 43:3014-3017.

[6] Imamura, N., Nishuima, M., Adachi, K. Novel antimycin antibiotics, Urauchimycins A and B produced by marine actionmycete. J. Antibiotics, 1993, 46:241-246.

[7] Pullen,C., Schmitz,P., Meurer,K. et al.New and bioactive compounds from Streptomyces strains residing in the wood of Celastraceae.Planta,2002,216:162-167. 